\title{
The theory of incoherent scattering of synchrotron radiation by vibrating nuclei in a crystal
}

\author{
V G Kohn ${ }^{1}$ and A I Chumakov ${ }^{1,2}$ \\ ${ }^{1}$ Russian Research Centre 'Kurchatov Institute', 123182 Moscow, Russia \\ ${ }^{2}$ European Synchrotron Radiation Facility, BP-220, 38043 Grenoble, France \\ E-mail: chumakov@esrf.fr
}

Received 16 May 2002

Published 1 November 2002

Online at stacks.iop.org/JPhysCM/14/11875

\begin{abstract}
The theory of resonant scattering of synchrotron radiation by vibrating nuclei in a crystal lattice with absorption or emission of phonons is developed under the conditions of incoherent scattering of x-rays over various nuclei. Consistent with previous works, we use the traditional approach based on Maxwell's equations and time-dependent quantum mechanics. A concept of coherence in scattering processes, properties of synchrotron radiation and conditions of measurements are analysed. The equations for energy dependence and time evolution of scattered radiation are derived in detail in the case of the nonsplit nuclear levels. The effect of hyperfine splitting on time evolution is also discussed. The time evolution of the scattered radiation soon after the primary flash depends on the temporal properties of the monochromator and has a more complicated form than the exponential nuclear decay. Later, the monochromator does not influence the time evolution. The energy dependence of space-integrated nuclear inelastic scattering is analogous to that of nuclear inelastic absorption.
\end{abstract}

\section{Introduction}

Nuclear resonant scattering of x-rays attracted much interest after Mossbauer's discovery of elastic resonant scattering by nuclei in a crystal [1], where a recoil energy is transferred to an entire crystal lattice, and the frequency of scattered radiation remains resonant. It gave access to studies of coherent phenomena in nuclear scattering and stimulated the development of the theory of coherent elastic nuclear resonant scattering (see the review of theoretical results in $[2,3])$. It soon became clear after the discovery that nuclear resonant scattering also gives a good opportunity to study the dynamics of nuclei in a crystal, especially diffusion and thermal vibrations [4-6]. The reason is the very small energy width of nuclear resonance. The measurements of phonon spectra with radioactive sources, however, were extremely difficult due to a small cross-section of inelastic processes. 
Recently these studies became feasible due to the development of third generation synchrotron radiation sources such as ESRF (France), APS (USA) and SPring8 (Japan). The high brightness and spectral density of these sources on the one hand, and the development of x-ray monochromators with an energy bandwidth of about $1 \mathrm{meV}$ on the other, gave rise to numerous experimental studies of nuclear resonant inelastic absorption. In such experiments one measures the atomic fluorescence, which results from the resonant absorption of incident photons by the nucleus with simultaneous absorption or emission of phonons in solids. The review of the experimental works and details of the experimental methods are discussed in [7]. The analysis of the early experimental results was based on the theory developed by Singwi and Sjölander [5]. This theory was, strictly speaking, formulated only for the particular case of Bravais cubic crystal lattices. The theory for anisotropic crystals was developed later [8].

In those works, only part of the nuclear cross-section related to the absorption of a photon by a vibrating nucleus was considered. The incident radiation was approximated by a monochromatic wave, i.e., spectral properties of synchrotron radiation and time-gating conditions of measurements were not considered. The relevance of the applied approximations to real experimental conditions was not analysed. As for the theory of nuclear resonant inelastic scattering (nuclear fluorescence), it was presented recently [9] using quantum electrodynamics and Green functions. This approach is rather difficult and requires special theoretical background. Moreover, the complicated mathematics do not allow an explicit description of scattering processes.

Another approach is widely used for the description of various processes of x-ray scattering after Laue's work [10]. It is based on Maxwell's equations and the dielectric properties of a medium. In some works (e.g., [2]) nuclear resonant scattering is described by Maxwell's equations, whereas a source of scattered radiation is treated as nuclear current. The latter is calculated by means of the time dependent perturbation theory of quantum mechanics [11]. We present the theory of incoherent nuclear resonant inelastic scattering which is developed within this conventional approach. It allows us to explicitly take into account the properties of synchrotron radiation as well as conditions of measurements. The theory is applicable for a description of experiments like the one performed in [12]. We verify that the measurement of incoherent inelastic nuclear resonant scattering in solids allows one to reconstruct the density of phonon state in the same manner as the inelastic nuclear absorption.

\section{Solution for incident and scattered waves}

\subsection{Approximation of incoherent scattering}

Very generally speaking, all scattering processes are coherent. For x-rays with an energy of about $10 \mathrm{keV}$ and more, the amplitudes of electric $\boldsymbol{E}(\boldsymbol{r}, t)$ and magnetic $\boldsymbol{B}(\boldsymbol{r}, t)$ fields are strictly connected to each other, and their modulo squared values are identical after averaging over a period of oscillations. Therefore, it is sufficient to consider an equation for any of the fields, or for some vector, which allows one to determine both of them. In the quantum theory of radiation (see, for example, [13]), the vector potential $\boldsymbol{A}(\boldsymbol{r}, t)$ in the Coulomb gauge is used for this purpose. Maxwell's equation for the vector potential can be written as

$$
\nabla^{2} \boldsymbol{A}(\boldsymbol{r}, t)-\frac{1}{c^{2}} \frac{\partial^{2}}{\partial t^{2}} \boldsymbol{A}(\boldsymbol{r}, t)=-\frac{1}{c} \boldsymbol{j}(\boldsymbol{r}, t),
$$

where $j(r, t)$ is the current acting as a source of electromagnetic radiation, and $c$ is the speed of light. The current $j(\boldsymbol{r}, t)$ results from moving charged particles. If the particles move independently of radiation, they are regarded as free sources. If the motion of particles is 
forced by incident radiation, we are dealing with re-radiation or scattering. In the latter case, the induced current is proportional to the radiation field strength.

In free space (without sources or scatterers), Maxwell's equation describes coherent propagation of the field having accurate phase relations between various points. The current, induced by x-rays, depends linearly on the field strength with high accuracy. Therefore, the phase relations are preserved, and Maxwell equations in matter describe the coherent scattering. The appearance of scattered waves can lead to numerous interference phenomena, where the intensity of the wave field becomes re-distributed in space and time. In addition, if the scattering probability is essentially high, the multiple-scattering processes become significant. The interference in multiple scattering leads to refraction, change of the propagation speed and other dynamical phenomena such as the acceleration of nuclear decay and the enhancement of the radiative channel in nuclear elastic forward or Bragg scattering [2].

However, the intrinsic coherence of scattering does not necessarily result in interference phenomena. The possibility to observe interference is determined by the conditions of measurement. First of all, independent sources of radiation produce waves, which phases do not correlate in space and time. Though the intensity of radiation from different sources is always positive and must be added only, the interference term in the modulo squared of total field strength has alternating signs in various time-space regions. As a rule, it oscillates over space and time and its average value vanishes. The same holds for the case of weak scattering, where the dynamical effects due to multiple scattering are negligible. Under these conditions, the coherent phenomena (determined by the non-vanishing interference term) can be revealed only with measurements having essentially a high spatial and/or temporal resolution. In contrast, if one deals with measurements of single scattering integrated over space and/or time, the results cannot show coherent phenomena.

For example, nuclear resonant scattering via different nuclear levels creates waves with a very large differences in frequencies. It is impossible to record the interference term even with the modern techniques. Therefore, such scattering events should be considered as incoherent (see the discussion in [9]). However, scattering via different sub-levels of hyperfine structure of the same nuclear level leads to quantum beats with a period of about $10 \mathrm{~ns}$. Time resolution of available detectors allows measurements of resulting interference (see the review of experimental results in [14]). These quantum beats are a result of coherent scattering.

Similar situations take place for the distribution in space. For example, if a source is strongly localized and a detector has sufficiently high spatial resolution, one can expect to record the interference phenomena even with x-rays of high energy [15]. In general, the concept of coherence can be formulated as a possibility to observe the interference over various sources and/or scatterers.

In this work, we consider nuclear resonant scattering accompanied by absorption or emission of phonons in a crystal. The process has a relatively small cross-section with a characteristic multiple-scattering length of several metres, much larger than the typical sample size. Therefore, we neglect multiple scattering and the related dynamical effects. In the absence of multiple scattering, a coherent superposition of waves from various scatterers can merely re-distribute the wave field in space, without changing its average intensity. We will, however, be interested in the angular-integral characteristics of scattering, where the interference effects vanish.

Thus, we can a priori restrict ourselves to the analysis of incoherent scattering. We use the first Born approximation for scattering by a single nucleus and consider a sum of intensities of waves scattered by all nuclei through a trivial factor of the nuclei density in the crystal. We solve the problem in three steps. Firstly, we discuss the properties of the incident radiation $\boldsymbol{A}_{i}(\boldsymbol{r}, t)=\boldsymbol{e}_{i} A_{i}(\boldsymbol{r}, t)$. Secondly, we calculate the current $\boldsymbol{j}_{f i}(\boldsymbol{r}, t)$ of nuclear 
resonant transitions. Finally, we calculate the scattered radiation $\boldsymbol{A}_{f}(\boldsymbol{r}, t)=e_{f} A_{f}(\boldsymbol{r}, t)$, at a large distance from the nucleus. Here $e_{i}$ and $e_{f}$ are the unit polarization vectors.

\subsection{Properties of incident radiation}

Properties of synchrotron undulator radiation are well known. They are complicated by the significant relativistic phenomena. Instead of exact analysis, we consider the simplified model, where the source current is strictly localized in time and space.

$$
\nabla^{2} A_{i}(\boldsymbol{r}, t)-\frac{1}{c^{2}} \frac{\partial^{2}}{\partial t^{2}} A_{i}(\boldsymbol{r}, t)=-\frac{1}{c} \delta\left(\boldsymbol{r}-\boldsymbol{r}_{0}\right) \delta\left(t-t_{s}\right)
$$

Here $\boldsymbol{r}_{0}$ is the source position, and $t_{s}$ is the instant of flash. Although a bunch of electrons in a storage ring consists of many electrons, the radiation from various electrons can be considered as incoherent. Therefore, each event of nuclear scattering is initiated by incident radiation from a single electron. The width of the synchrotron radiation pulse (e.g., about $100 \mathrm{ps}$ at the ESRF) enters the problem through the final convolution of the scattered intensity with the pulse shape (this point will not be considered here).

The solution of equation (2) can be obtained as the Fourier integral

$$
A_{i}(\boldsymbol{r}, t)=\int \frac{\mathrm{d} \omega}{2 \pi} \exp \left(-\mathrm{i} \omega\left[t-t_{s}\right]\right) \frac{\exp \left(\mathrm{i} \omega\left|\boldsymbol{r}-\boldsymbol{r}_{0}\right| / c\right)}{4 \pi c\left|\boldsymbol{r}-\boldsymbol{r}_{0}\right|},
$$

where the particular solution for the monochromatic source has the shape of a spherical wave. We note that the integral in (3) can be calculated analytically as follows:

$$
A_{i}(\boldsymbol{r}, t)=\frac{\delta\left(t-t_{s}-\left|\boldsymbol{r}-\boldsymbol{r}_{0}\right| / c\right)}{4 \pi c\left|\boldsymbol{r}-\boldsymbol{r}_{0}\right|} .
$$

This result has a simple physical meaning. Instantaneous localized excitation spreads in all directions from the point $\boldsymbol{r}_{0}$ and is distributed over the sphere surface with radius $\left|\boldsymbol{r}-\boldsymbol{r}_{0}\right|$. At the instant $t$, the radius is equal to the distance $\left|\boldsymbol{r}-\boldsymbol{r}_{0}\right|=c\left(t-t_{s}\right)$ covered by light during the time interval $\left(t-t_{s}\right)$. The intensity of the radiation is proportional to $\left|r-r_{0}\right|^{-2}$, so that the total intensity over the sphere area remains constant.

In further calculations, the integral presentation (3) is more convenient. At long distances from the source $\left|\boldsymbol{r}_{0}\right| \gg|\boldsymbol{r}|$, we can use the approximation

$$
A_{i}(\boldsymbol{r}, t) \approx \frac{1}{4 \pi c\left|\boldsymbol{r}_{0}\right|} \int \frac{\mathrm{d} \omega}{2 \pi} \exp \left(\mathrm{i} \boldsymbol{k}_{0}^{\prime} \boldsymbol{r}-\mathrm{i} \omega\left[t-t_{s}-t_{0}\right]\right),
$$

where $t_{0}=\left|\boldsymbol{r}_{0}\right| / c$ is the flight time through the distance $\left|\boldsymbol{r}_{0}\right|$ from the source to the origin of the coordinate system where the nucleus is located, $\boldsymbol{k}_{0}^{\prime}=(\omega / c) \boldsymbol{s}_{0}, \boldsymbol{s}_{0}=-\boldsymbol{r}_{0} /\left|\boldsymbol{r}_{0}\right|$. Here we applied the approximation $\left|\boldsymbol{r}-\boldsymbol{r}_{0}\right| \approx\left|\boldsymbol{r}_{0}\right|+\boldsymbol{s}_{0} \boldsymbol{r}$. The expression (5) describes the set of plane monochromatic waves with all the possible frequencies.

In reality, the electron in the storage ring is not localized in space but moves almost at the speed of light. Therefore, synchrotron radiation is strongly anisotropic. It is close to the spherical wave only in the electron coordinate system $[16,17]$. In the laboratory coordinate system, it can be approximated at distant points by the Gaussian distribution centred on the direction of the electron beam. Within its central part, however, the radiation wave field is close to the spherical wave. Thus, the postulated spatial localization is consistent with experimental conditions. In order to estimate the temporal localization of the radiation flash from the single electron in the laboratory coordinate system, we can consider the effective duration of the radiation flash as given by the frequency spectrum of synchrotron radiation and the uncertainty principle. It is negligibly small compared to the nuclear lifetime. Thus, 
equation (3) is approximately consistent with the properties of synchrotron radiation under the typical experimental conditions (see, for example, [12]).

In experiments on nuclear inelastic scattering, the bandwidth of radiation is limited by a monochromator. Let the transmission amplitude of the monochromator be $P\left(\omega-\omega_{0}\right)$, where $\omega_{0}$ is the frequency at the maximum of the monochromator throughput. The instrumental function $I_{M}\left(\omega^{\prime}\right)=\left|P\left(\omega^{\prime}\right)\right|^{2}$ has a maximum at the zero relative frequency $\omega^{\prime}=\omega-\omega_{0}$ and provides the frequency band $\Delta \omega$. We neglect the frequency dependence of the wavevector $\boldsymbol{k}_{0}^{\prime}$. This can be done if $\Delta \omega|\boldsymbol{r}| / c<\pi$, where the vector $|\boldsymbol{r}|$ is determined by the thermal displacements of the nucleus. The typical value of the monochromator energy bandwidth is $\hbar \Delta \omega=1 \mathrm{meV}$ (see, for example, [7]). The typical value of $|r|$ is $0.1 \AA$. Taking this into account we obtain an estimation $\Delta \omega|r| / c \approx 0.5 \times 10^{-7} \ll 1$. Thus we arrive at the following approximation for the amplitude of the incident radiation:

$A_{i}(\boldsymbol{r}, t) \approx \frac{\exp \left(\mathrm{i} \boldsymbol{k}_{0} \boldsymbol{r}-\mathrm{i} \omega_{0}\left[t-t_{s}-t_{0}\right]\right)}{4 \pi c\left|\boldsymbol{r}_{0}\right|} \int \frac{\mathrm{d} \omega^{\prime}}{2 \pi} \exp \left(-\mathrm{i} \omega^{\prime}\left[t-t_{s}-t_{0}\right]\right) P\left(\omega^{\prime}\right)$.

Hereafter $\boldsymbol{k}_{0}=\left(\omega_{0} / c\right) \boldsymbol{s}_{0}$.

The frequency integral in (6) describes time evolution of the incident radiation flash after passing through the monochromator. It is determined by the response function of the monochromator

$$
\tilde{P}(t)=\int \frac{\mathrm{d} \omega}{2 \pi} \exp (-\mathrm{i} \omega t) P(\omega) .
$$

According to general physical principles, $P(\omega)$ as a function of complex variable $\omega$ can have poles only in the bottom half-plane. Therefore, the response function $\tilde{P}(t)$ vanishes for $t<0$. In other words, it describes the retarded response. The nucleus perceives the radiation flush at the instant $t=t_{s}+t_{0}$, which is later than $t_{s}$ - the instant of the flash at the source-by $t_{0}$. Time $t_{0}$ is required for light to travel the distance $\left|\boldsymbol{r}_{0}\right|$. The exact time profile of the flash perceived by the nucleus is determined by the monochromator and is described by the function $\tilde{P}(t)$.

\subsection{Structure of scattered wave}

It will be shown below that the current of nuclear transitions can be expressed as an integral over a limited range of frequencies near the middle frequency $\omega_{1}$,

$$
\boldsymbol{e}_{f} \boldsymbol{j}_{f i}(\boldsymbol{r}, t)=\int \frac{\mathrm{d} \omega^{\prime}}{2 \pi} \exp \left[-\mathrm{i}\left(\omega_{1}+\omega^{\prime}\right) t\right] J\left(\boldsymbol{r}, \omega^{\prime}\right) .
$$

Therefore, we can also write the amplitude of the scattered wave at the detector position as a spherical monochromatic wave:

$A_{f}\left(\boldsymbol{r}_{1}, t_{d}\right)=\int \frac{\mathrm{d} \omega^{\prime}}{2 \pi} \exp \left[-\mathrm{i}\left(\omega_{1}+\omega^{\prime}\right) t_{d}\right] \int \mathrm{d} \boldsymbol{r} \frac{\exp \left(\mathrm{i} k_{1}^{\prime}\left|\boldsymbol{r}_{1}-\boldsymbol{r}\right|\right)}{4 \pi c\left|\boldsymbol{r}_{1}-\boldsymbol{r}\right|} J\left(\boldsymbol{r}, \omega^{\prime}\right)$,

where $t_{d}$ is the instant the radiation flash hits the detector, and $k_{1}^{\prime}=\left(\omega_{1}+\omega^{\prime}\right) / c$.

We assume that the distance $\left|\boldsymbol{r}_{1}\right|$ between the detector and the scatterer is much larger than the typical displacement of the nucleus $|r|$ due to thermal vibrations. We again place the centre of the scatterer at the origin of the coordinate system and use the relation $\boldsymbol{r}_{1}-\boldsymbol{r}=\left|\boldsymbol{r}_{1}\right| \boldsymbol{s}_{1}-\boldsymbol{r}$, where $s_{1}=r_{1} /\left|\boldsymbol{r}_{1}\right|$. Then, applying an approximation $\left|\boldsymbol{r}_{1}-\boldsymbol{r}\right| \approx\left|\boldsymbol{r}_{1}\right|-\boldsymbol{s}_{1} \boldsymbol{r}$, we arrive at the expression

$A_{f}\left(\boldsymbol{r}_{1}, t_{d}\right)=\int \frac{\mathrm{d} \omega^{\prime}}{2 \pi} \frac{\exp \left[-\mathrm{i}\left(\omega_{1}+\omega^{\prime}\right)\left(t_{d}-t_{1}\right)\right]}{4 \pi c\left|\boldsymbol{r}_{1}\right|} \int \mathrm{d} \boldsymbol{r} \exp \left(-\mathrm{i} \boldsymbol{k}_{1} \boldsymbol{r}\right) J\left(\boldsymbol{r}, \omega^{\prime}\right)$.

Here $\boldsymbol{k}_{1}$ is the scattering vector, $\boldsymbol{k}_{1}=k_{1} \boldsymbol{s}_{1}=k_{1} \boldsymbol{r}_{1} /\left|\boldsymbol{r}_{1}\right|$. Its modulus is $k_{1}=\omega_{1} / c$, because we can neglect the small frequency $\omega^{\prime}$ for the same reason as in the case of incident radiation. 
The value $t_{1}=\left|\boldsymbol{r}_{1}\right| / c$ is the time interval required for light to travel the distance $\left|\boldsymbol{r}_{1}\right|$ from the nucleus to the detector. Thus, the scattered radiation from the single nucleus is described by an anisotropic spherical wave, whose shape is determined by the properties of the nucleus.

\section{Quantum current of nuclear transitions}

The scatterer is the nucleus located at a node of the crystal lattice in the target. The state of the scatterer can be described in terms of the collective state of the centres of mass of all nuclei in the crystal $\left|\chi_{i}\right\rangle$ and the internal states of the nucleus $\left|\phi_{i}\right\rangle$. Consider first the internal nuclear transitions. The nuclear current $j(r, t)$ is the quantum mechanical average value of the current density operator over the states in the presence of the radiation electromagnetic field. The resonant scattering is described by the transition of second order from the initial state $\left|\phi_{i}\right\rangle$ into the final state $\left|\phi_{f}\right\rangle$ assuming that the transition proceeds through the intermediate state $\left|\phi_{j}\right\rangle$. In accordance with the time-dependent perturbation theory of quantum mechanics we have

$$
\begin{gathered}
\boldsymbol{j}_{f i}(\boldsymbol{r}, t)=\frac{\exp \left(\mathrm{i} \omega_{f i}^{(n)} t\right)}{\mathrm{i} \hbar} \int \frac{\mathrm{d} \boldsymbol{k}}{(2 \pi)^{3}} \int_{-\infty}^{t} \mathrm{~d} t^{\prime} \mathrm{e}^{\mathrm{i} k\left[r-r_{n}(t)\right]}\left\langle\phi_{f}\left|\hat{\boldsymbol{j}}_{n}(\boldsymbol{k})\right| \phi_{j}\right\rangle \\
\times\left\langle\phi_{j}\left|\hat{H}_{i n t}\left(t^{\prime}\right)\right| \phi_{i}\right\rangle \exp \left[\mathrm{i}\left(-\omega_{j i}^{(n)}+\mathrm{i} \gamma\right)\left(t-t^{\prime}\right)\right]
\end{gathered}
$$

where $\boldsymbol{r}_{n}(t)$ is the time-dependent coordinate of the vibrating nucleus, $\omega_{i j}^{(n)}=\omega_{i}^{(n)}-\omega_{j}^{(n)}$, $\hbar \omega_{l}^{(n)}$ is the energy level of the $l$ th nuclear state, $l=i, j, f ; \gamma=\Gamma / 2 \hbar$ and $\Gamma$ is the energy width of the intermediate state $\left|\phi_{j}\right\rangle$. The index $(n)$ is related to the nuclear transitions.

The interaction Hamiltonian is well known. It is convenient to write it in the form

$$
\begin{aligned}
\hat{H}_{\text {int }}\left(t^{\prime}\right)=- & \frac{1}{c} \int \mathrm{d} \boldsymbol{r}^{\prime} \hat{\boldsymbol{j}}_{n}\left(\boldsymbol{r}^{\prime}-\boldsymbol{r}_{n}\left(t^{\prime}\right)\right) \boldsymbol{A}_{i}\left(\boldsymbol{r}^{\prime}, t^{\prime}\right) \\
& =-\frac{1}{c} \int \mathrm{d} \boldsymbol{r}^{\prime} \int \frac{\mathrm{d} \boldsymbol{k}^{\prime}}{(2 \pi)^{3}} \mathrm{e}^{\mathrm{i} \boldsymbol{k}^{\prime}\left(\boldsymbol{r}^{\prime}-\boldsymbol{r}_{n}\left(t^{\prime}\right)\right)} \boldsymbol{e}_{i} \hat{\boldsymbol{j}}_{n}\left(\boldsymbol{k}^{\prime}\right) A_{i}\left(\boldsymbol{r}^{\prime}, t^{\prime}\right),
\end{aligned}
$$

where, as above, $\boldsymbol{e}_{i}$ is the unit vector of polarization of incident radiation. Then, we obtain the projection of the current density on the unit vector of polarization of the scattered wave as

$$
\boldsymbol{e}_{f} \boldsymbol{j}_{f i}(\boldsymbol{r}, t)=\int_{-\infty}^{t} \mathrm{~d} t^{\prime} \int \mathrm{d} \boldsymbol{r}^{\prime} M_{f i}\left(\boldsymbol{r}, t ; \boldsymbol{r}^{\prime}, t^{\prime}\right) A_{i}\left(\boldsymbol{r}^{\prime}, t^{\prime}\right),
$$

where we introduce the scattering matrix

$$
\begin{aligned}
M_{f i}\left(\boldsymbol{r}, t ; \boldsymbol{r}^{\prime}, t^{\prime}\right) & =\frac{\mathrm{i} \exp \left(\mathrm{i} \omega_{f i}^{(n)} t\right)}{\hbar c} \int \frac{\mathrm{d} \boldsymbol{k} \mathrm{d} \boldsymbol{k}^{\prime}}{(2 \pi)^{6}} \mathrm{e}^{\mathrm{i} k r+\mathrm{i} k^{\prime} \boldsymbol{r}^{\prime}} N_{f i}\left(\boldsymbol{k}, \boldsymbol{k}^{\prime}\right) \\
& \times \exp \left[\mathrm{i}\left(-\omega_{j i}^{(n)}+\mathrm{i} \gamma\right)\left(t-t^{\prime}\right)\right] \mathrm{e}^{-\mathrm{i} k r_{n}(t)} \mathrm{e}^{-\mathrm{i} k^{\prime} r_{n}\left(t^{\prime}\right)} .
\end{aligned}
$$

Hereafter we use the notation

$$
N_{f i}\left(\boldsymbol{k}, \boldsymbol{k}^{\prime}\right)=\left\langle\phi_{f}\left|\boldsymbol{e}_{f} \hat{\boldsymbol{j}}_{n}(\boldsymbol{k})\right| \phi_{j}\right\rangle\left\langle\phi_{j}\left|\boldsymbol{e}_{i} \hat{\boldsymbol{j}}_{n}\left(\boldsymbol{k}^{\prime}\right)\right| \phi_{i}\right\rangle .
$$

Expression (14) for the scattering matrix contains the coordinates of the nucleus at the instant of excitation $t^{\prime}$ and at the instant of relaxation $t$.

The nuclear coordinates depend on time owing to thermal vibrations. Thermal vibrations of atoms in a crystal lattice exhibit distinct quantum behaviour. Therefore they must be described as absorption and emission of phonons. We consider the coordinate $\boldsymbol{r}_{n}$ as the operator and introduce the wavefunction of phonons.

$$
\begin{gathered}
\mathrm{e}^{-\mathrm{i} k r_{n}(t)} \mathrm{e}^{-\mathrm{i} \boldsymbol{k}^{\prime} \boldsymbol{r}_{n}\left(t^{\prime}\right)}=\sum_{m}\left\langle\chi_{f}\left|\mathrm{e}^{-\mathrm{i} k r_{n}}\right| \chi_{m}\right\rangle\left\langle\chi_{m}\left|\mathrm{e}^{-\mathrm{i} k^{\prime} r_{n}}\right| \chi_{i}\right\rangle \exp \left(\mathrm{i} \omega_{f m}^{(p)} t+\mathrm{i} \omega_{m i}^{(p)} t^{\prime}\right) \\
=\exp \left(\mathrm{i} \omega_{f i}^{(p)} t\right) L_{f i}\left(\boldsymbol{k}, \boldsymbol{k}^{\prime}, t-t^{\prime}\right)
\end{gathered}
$$


Here $(p)$ indicates the frequency related to the phonon system. The sum over the intermediate states of the phonon system is required in order to describe an evolution of the system from the instant $t^{\prime}$ into the instant $t$. The function $L_{f i}$ depends only on the time interval and is defined by the expression

$$
L_{f i}\left(\boldsymbol{k}, \boldsymbol{k}^{\prime}, t\right)=\sum_{m}\left\langle\chi_{f}\left|\mathrm{e}^{-\mathrm{i} k r_{n}}\right| \chi_{m}\right\rangle\left\langle\chi_{m}\left|\mathrm{e}^{-\mathrm{i} k^{\prime} r_{n}}\right| \chi_{i}\right\rangle \exp \left(-\mathrm{i} \omega_{m i}^{(p)} t\right) .
$$

Then the scattering matrix takes the form of

$$
\begin{aligned}
M_{f i}\left(\boldsymbol{r}, t ; \boldsymbol{r}^{\prime}, t^{\prime}\right) & =\frac{\mathrm{i} \exp \left(\mathrm{i} \omega_{f i} t\right)}{\hbar c} \exp \left[\mathrm{i}\left(-\omega_{r}+\mathrm{i} \gamma\right)\left(t-t^{\prime}\right)\right] \\
& \times \int \frac{\mathrm{d} \boldsymbol{k} \mathrm{d} \boldsymbol{k}^{\prime}}{(2 \pi)^{6}} \mathrm{e}^{\mathrm{i} \boldsymbol{k} r+\mathrm{i} \boldsymbol{k}^{\prime} \boldsymbol{r}^{\prime}} N_{f i}\left(\boldsymbol{k}, \boldsymbol{k}^{\prime}\right) L_{f i}\left(\boldsymbol{k}, \boldsymbol{k}^{\prime}, t-t^{\prime}\right),
\end{aligned}
$$

where we introduce the total transition frequency $\omega_{f i}=\omega_{f i}^{(n)}+\omega_{f i}^{(p)}$ and the resonance frequency $\omega_{r}=\omega_{j i}^{(n)}$. Expression (18) is close to equation (25) of the work [9]. However, here it is derived for a three-level system within the standard quantum mechanics approach. A sum over the intermediate states of the nucleus, made in [9], does not appear here, because the transition proceeds through the only intermediate state.

Inserting (18) and (6) into (13) and using the transformations discussed above, we obtain the explicit form for the function $J(r, \omega)$ in (8) as follows:

$$
\begin{gathered}
J(\boldsymbol{r}, \omega)=P(\omega) \frac{\mathrm{i} \exp \left[\mathrm{i}\left(\omega_{0}+\omega\right)\left(t_{s}+t_{0}\right)\right]}{4 \pi \hbar c^{2}\left|\boldsymbol{r}_{0}\right|} \int \frac{\mathrm{d} \boldsymbol{k}}{(2 \pi)^{3}} \exp (\mathrm{i} \boldsymbol{k} \boldsymbol{r}) N_{f i}\left(\boldsymbol{k},-\boldsymbol{k}_{0}\right) \\
\times \tilde{L}_{f i}\left(\boldsymbol{k},-\boldsymbol{k}_{0}, \omega_{0}+\omega-\omega_{r}+\mathrm{i} \gamma\right) .
\end{gathered}
$$

Here $\omega_{1}=\omega_{0}-\omega_{f i}$ is the middle frequency of the scattered wave. We have also introduced the function

$$
\tilde{L}_{f i}\left(\boldsymbol{k}, \boldsymbol{k}^{\prime}, \omega\right)=\int_{0}^{\infty} \mathrm{d} t \exp (\mathrm{i} \omega t) L_{f i}\left(\boldsymbol{k}, \boldsymbol{k}^{\prime}, t\right)
$$

\section{Properties of scattered radiation}

Inserting (19) in (10), we obtain the vector potential of the scattered wave as follows:

$$
\begin{aligned}
A_{f}\left(\boldsymbol{r}_{1}, t_{d}\right)= & \frac{\exp \left[-\mathrm{i} \omega_{1}\left(t_{d}-t_{1}\right)\right] \exp \left[\mathrm{i} \omega_{0}\left(t_{s}+t_{0}\right)\right]}{(4 \pi)^{2} c^{3} \hbar\left|\boldsymbol{r}_{0}\right|\left|\boldsymbol{r}_{1}\right|} N_{f i}\left(\boldsymbol{k}_{1},-\boldsymbol{k}_{0}\right) \\
& \times \int \frac{\mathrm{d} \omega}{2 \pi} \exp (-\mathrm{i} \omega t) \tilde{L}_{f i}\left(\boldsymbol{k}_{1},-\boldsymbol{k}_{0}, \omega_{0}+\omega-\omega_{r}+\mathrm{i} \gamma\right) P(\omega) .
\end{aligned}
$$

Here $t=t_{d}-t_{s}-t_{0}-t_{1}$ is the time delay of radiation in the monochromator and in the nucleus, because $t_{d}$ is the time when the scattered flash hits the detector, whereas $\left(t_{s}+t_{0}+t_{1}\right)$ would be that for radiation travelling along the same trajectory in vacuum. The definition (20) states that the function $\tilde{L}_{f i}\left(\boldsymbol{k}_{1},-\boldsymbol{k}_{0}, \omega\right)$ has poles only in the bottom half-plane of the complex variable $\omega$. Therefore, the strength of the scattered wave as a function of the delay time $t$ vanishes for $t<0$. The general expression for the intensity of scattered radiation as a function of the delay time $t$ and the mean frequency $\omega_{0}$ of the monochromator throughput is

$I\left(\omega_{0}, t\right)=I_{0} I_{N}\left|\int \frac{\mathrm{d} \omega}{2 \pi} \exp (-\mathrm{i} \omega t) \tilde{L}_{f i}\left(\boldsymbol{k}_{1},-\boldsymbol{k}_{0}, \omega_{0}+\omega-\omega_{r}+\mathrm{i} \gamma\right) P(\omega)\right|^{2}$,

where

$$
I_{0}=\frac{\omega_{1}^{2}}{(4 \pi)^{4} c^{8} \hbar^{2} r_{0}^{2} r_{1}^{2}}, \quad I_{N}=\left|N_{f i}\left(\boldsymbol{k}_{1},-\boldsymbol{k}_{0}\right)\right|^{2}
$$


In measurements of nuclear inelastic scattering, the signal is accumulated over many pulses of synchrotron radiation and results from scattering by many nuclei. Therefore, the above expression must be summed over the final states (index $f$ ) and averaged over the initial states (index $i$ ) of the phonon system. We will denote such an intensity as $\overline{I\left(\omega_{0}, t\right)}$ and call it the average intensity.

\subsection{Energy dependence of scattered radiation}

It is interesting to first analyse the energy dependence of the scattered radiation, when it is collected over an entire time interval from zero to infinity. The time-integral intensity is

$$
I_{\text {int }}\left(\omega_{0}\right)=\int_{0}^{\infty} I\left(\omega_{0}, t\right) \mathrm{d} t
$$

Since $I(t)=0$ at $t<0$, the integration limits can be formally replaced by $(-\infty, \infty)$. Taking into account that expression (22) is the modulo squared of the Fourier integral over frequency, we use Parseval's theorem and obtain

$$
I_{\text {int }}\left(\omega_{0}\right)=I_{0} I_{N} \int \frac{\mathrm{d} \omega}{2 \pi}\left|\tilde{L}_{f i}\left(\boldsymbol{k}_{1},-\boldsymbol{k}_{0}, \omega_{0}-\omega_{r}+\omega+\mathrm{i} \gamma\right)\right|^{2} I_{M}(\omega) .
$$

Thus, the time-integrated intensity of scattered radiation as a function of the middle frequency $\omega_{0}$ of incident radiation is a convolution of the energy dependence of inelastic scattering of the monochromatic wave with the instrumental function of the monochromator $I_{M}(-\omega)=$ $|P(-\omega)|^{2}$.

In order to calculate the average intensity $\overline{I_{i n t}\left(\omega_{0}\right)}$, we have to average the function $\left|\tilde{L}_{f i}\left(\boldsymbol{k}_{1},-\boldsymbol{k}_{0}, \omega+\mathrm{i} \gamma\right)\right|^{2}$. Inserting (17) in (20), we obtain

$$
\tilde{L}_{f i}\left(\boldsymbol{k}_{1},-\boldsymbol{k}_{0}, \omega+\mathrm{i} \gamma\right)=-\sum_{m} \frac{\left\langle\chi_{f}\left|\mathrm{e}^{-\mathrm{i} k_{1} r_{n}}\right| \chi_{m}\right\rangle\left\langle\chi_{m}\left|\mathrm{e}^{\mathrm{i} k_{0} r_{n}}\right| \chi_{i}\right\rangle}{\left(\omega-\omega_{m i}^{(p)}+\mathrm{i} \gamma\right)} .
$$

The modulo squared right-hand side must be summarized over the final states. This leads to the expression

$$
\sum_{f, m, m^{\prime}} \frac{\left\langle\chi_{f}\left|\mathrm{e}^{-\mathrm{i} k_{1} r_{n}}\right| \chi_{m}\right\rangle\left\langle\chi_{m}\left|\mathrm{e}^{\mathrm{i} k_{0} r_{n}}\right| \chi_{i}\right\rangle}{\left(\omega-\omega_{m i}^{(p)}+\mathrm{i} \gamma\right)} \frac{\left\langle\chi_{i}\left|\mathrm{e}^{-\mathrm{i} k_{0} r_{n}}\right| \chi_{m^{\prime}}\right\rangle\left\langle\chi_{m^{\prime}}\left|\mathrm{e}^{\mathrm{i} k_{1} r_{n}}\right| \chi_{f}\right\rangle}{\left(\omega-\omega_{m^{\prime} i}^{(p)}-\mathrm{i} \gamma\right)} .
$$

Here the sum over $f$ can be calculated independently as

$$
\sum_{f}\left\langle\chi_{m^{\prime}}\left|\mathrm{e}^{\mathrm{i} k_{1} r_{n}}\right| \chi_{f}\right\rangle\left\langle\chi_{f}\left|\mathrm{e}^{-\mathrm{i} k_{1} r_{n}}\right| \chi_{m}\right\rangle=\left\langle\chi_{m^{\prime}}|| \chi_{m}\right\rangle=\delta_{m m^{\prime}}
$$

Then the dependence on the direction of the scattered wave vanishes and, instead of the triple sum, we obtain only a sum over $m$.

Replacing the index $m \rightarrow f$, we present the average intensity in the form of

$$
\overline{I_{\text {int }}\left(\omega_{0}\right)}=I_{0} I_{N} \int \frac{\mathrm{d} \omega}{2 \pi} I_{S S}\left(\omega_{0}-\omega_{r}+\omega\right) I_{M}(\omega),
$$

where

$I_{S S}(\omega)=\left\langle\sum_{f}\left|\tilde{L}_{f i}\left(\boldsymbol{k}_{1},-\boldsymbol{k}_{0}, \omega+\mathrm{i} \gamma\right)\right|^{2}\right\rangle_{T}=\sum_{i, f} \frac{g_{i}\left|\left\langle\chi_{f}\left|\exp \left(\mathrm{i} \boldsymbol{k}_{0} \boldsymbol{r}_{n}\right)\right| \chi_{i}\right\rangle\right|^{2}}{\left(\omega_{f i}^{(p)}-\omega\right)^{2}+\gamma^{2}}$.

Here $\langle\cdots\rangle_{T}$ means the thermal averaging over the initial states, and $g_{i}$ is the weight of the $i$ th initial state of the phonon system.

Equation (29) is a standard form of taking into account the instrumental function (see, for example, $[8,18])$. Equation (30) for the integral intensity of scattered radiation coincides 
with the known expression for the cross-section of nuclear resonant absorption, derived by Singwi and Sjölander (see equation (1) in article [5]). An important property of this formula is that absorption or emission of phonons takes place simultaneously with resonant absorption of incident photon by nucleus, i.e., at the instant of nuclear excitation. The intensity of scattered radiation depends only on the wavevector $\boldsymbol{k}_{0}$, whereas the wavevector $\boldsymbol{k}_{1}$ does not influence the energy dependence. As follows from (28), this feature is a logical consequence of the procedure of summation over all possible final states of the phonon system. The treatment corresponds to the experimental conditions, where all scattered quanta are collected independently of their energy and direction of propagation. Should an experimental setup contain additional elements allowing the selection of scattered radiation with a particular energy, or if the scattered radiation were to be accepted within an essentially small solid angle, we would have to use some more complicated expressions instead of (21) with an explicit integration over frequency $\omega_{1}$. Besides that, we would have to use the theory of coherent scattering, i.e., we would have to take into account the interference of scattering over various nuclei. In general, the energy dependence of radiation scattered into some particular direction may differ from the averaged value.

However, under the conditions of complete averaging over the parameters of scattered radiation, the latter depends on the energy of incident radiation in the same manner as nuclear inelastic absorption. This theoretical result agrees with the experimental results of the work [12]. A reason for such a coincidence is that spontaneous emission of photons during de-excitation of a single nucleus does not depend on the resonance condition of preceding nuclear excitation. In particular, it does not depend on the phonon state, which has determined conditions of nuclear excitation. First, a nucleus is excited by an incident quantum with simultaneous phonon absorption or emission. The following evolution of the excited nucleus may vary, but it does not influence the dependence of detected radiation on the energy of incident radiation.

Following [5], we represent the function $I_{S S}(\omega)$ as an integral over time using, however, a slightly different way of derivation which is more useful for the discussion below. Let us write the expression (30) as follows:

$$
I_{S S}(\omega)=\sum_{i, f} g_{i}\left|\left\langle\chi_{f}\left|\exp \left(\mathrm{i} \boldsymbol{k}_{0} \boldsymbol{r}_{n}\right)\right| \chi_{i}\right\rangle\right|^{2} \tilde{R}\left(\omega_{f i}^{(p)}-\omega\right),
$$

where

$$
\tilde{R}(\omega)=\frac{1}{\left(\omega^{2}+\gamma^{2}\right)}=\int \mathrm{d} t \exp (\mathrm{i} \omega t) R(t) .
$$

Inserting the Fourier integral (32) in (31) we obtain

$$
I_{S S}(\omega)=\int \mathrm{d} t \exp (-\mathrm{i} \omega t) F\left(\boldsymbol{k}_{0}, t\right) R(t),
$$

where the time correlation function $F\left(\boldsymbol{k}_{0}, t\right)$ depends on the lattice vibrations and is determined by

$$
F\left(\boldsymbol{k}_{0}, t\right)=\sum_{i} g_{i} \sum_{f}\left\langle\chi_{i}\left|\exp \left(-\mathrm{i} \boldsymbol{k}_{0} \boldsymbol{r}_{n}\right)\right| \chi_{f}\right\rangle\left\langle\chi_{f}\left|\exp \left(\mathrm{i} \boldsymbol{k}_{0} \boldsymbol{r}_{n}\right)\right| \chi_{i}\right\rangle \exp \left(\mathrm{i} \omega_{f i}^{(p)} t\right)
$$

This expression can be transformed into

$$
\begin{gathered}
F\left(\boldsymbol{k}_{0}, t\right)=\sum_{i} g_{i}\left\langle\chi_{i}\left|\exp \left(-\mathrm{i} \boldsymbol{k}_{0} \boldsymbol{r}_{n}(0)\right) \exp \left(\mathrm{i} \boldsymbol{k}_{0} \boldsymbol{r}_{n}(t)\right)\right| \chi_{i}\right\rangle \\
=\left\langle\exp \left(-\mathrm{i} \boldsymbol{k}_{0} \boldsymbol{r}_{n}(0)\right) \exp \left(\mathrm{i} \boldsymbol{k}_{0} \boldsymbol{r}_{n}(t)\right)\right\rangle_{T}
\end{gathered}
$$

The function $R(t)$ can be calculated in explicit form

$$
R(t)=\frac{\exp (-\gamma|t|)}{2 \gamma}
$$


Finally we arrive at the expression

$$
\overline{I_{\text {int }}\left(\omega_{0}\right)}=I_{0} I_{N} \int \mathrm{d} t \exp \left(-\mathrm{i}\left[\omega_{0}-\omega_{r}\right] t\right) F\left(\boldsymbol{k}_{0}, t\right) R(t) \tilde{I}_{M}(t),
$$

where

$$
\tilde{I}_{M}(t)=\int \frac{\mathrm{d} \omega}{2 \pi} \exp (-\mathrm{i} \omega t) I_{M}(\omega)
$$

In the works $[8,18]$, similar expressions were used in order to obtain the phonon density of states from the energy dependence of nuclear inelastic absorption. Our results show that the density of states can be obtained identically from the energy dependence of incoherent nuclear inelastic scattering as well, if it is ideally integrated over time. Strictly speaking, an ideal integration over time cannot be reached in experiment, because, during a short period after a synchrotron radiation flash, an acquisition is gated against strong electronic scattering. In order to understand the possible influence of the gating on the energy dependence of inelastic scattering, one needs to consider the time dependence of scattered radiation.

According to equations (34) and (37), the dependence of scattered radiation on the frequency $\omega_{0}$ is defined by the energy transitions of the phonon system, with the energy transfer $\hbar\left(\omega_{0}-\omega_{r}\right)$ if the Fourier image of the instrumental function depends weakly on $t$. This takes place for monochromators with an essentially narrow bandwidth. In the opposite case, where the monochromator bandwidth is very large, we have $\tilde{I}_{M}(t) \approx I_{M} \delta(t)$, therefore $\overline{I_{\text {int }}\left(\omega_{0}\right)} \approx I_{0} I_{N} I_{M}$ does not depend on $\omega_{0}$. Let us consider an instructive example where the phonon system has only one frequency $\omega^{(p)}$. Then, according to (34), the time correlation function $F\left(\boldsymbol{k}_{0}, t\right)=\exp \left(\mathrm{i} \omega^{(p)} t\right) F_{0}$ and, neglecting the time dependence of $R(t)$, we obtain $\overline{I_{\text {int }}\left(\omega_{0}\right)}=(2 \gamma)^{-1} F_{0} I_{0} I_{N} I_{M}\left(\omega_{r}+\omega^{(p)}-\omega_{0}\right)$. Therefore, the energy dependence will have a single peak at the position $\omega_{0}=\omega_{r}+\omega^{(p)}$, and the structure of the peak will coincide with the instrumental function.

\subsection{Time dependence of scattered radiation}

Equation (22) for the intensity of scattered radiation can be rewritten as follows:

$$
I\left(\omega_{0}, t\right)=I_{0} I_{N}\left|Q_{f i}\left(\omega_{0}, t\right)\right|^{2},
$$

where

$$
Q_{f i}\left(\omega_{0}, t\right)=\int \frac{\mathrm{d} \omega}{2 \pi} \exp (-\mathrm{i} \omega t) \tilde{L}_{f i}\left(\boldsymbol{k}_{1},-\boldsymbol{k}_{0}, \omega_{0}-\omega_{r}+\omega+\mathrm{i} \gamma\right) P(\omega) .
$$

Inserting (20), we obtain

$$
Q_{f i}\left(\omega_{0}, t\right)=\int_{0}^{t} \mathrm{~d} t^{\prime} \exp \left(\mathrm{i}\left[\omega_{0}-\omega_{r}+\mathrm{i} \gamma\right] t^{\prime}\right) L_{f i}\left(\boldsymbol{k}_{1},-\boldsymbol{k}_{0}, t^{\prime}\right) \tilde{P}\left(t-t^{\prime}\right) .
$$

Here we use (7) and the condition $\tilde{P}(t)=0$ for $t<0$. As follows from (41), the intensity vanishes at $t=0$. Making a replacement of the integration variable $t^{\prime} \rightarrow t-t^{\prime}$ and using (17), we obtain

$$
\begin{aligned}
Q_{f i}\left(\omega_{0}, t\right)= & \mathrm{e}^{-\gamma t} \mathrm{e}^{\mathrm{i}\left(\omega_{0}-\omega_{r}\right) t} \sum_{m}\left\langle\chi_{f}\left|\mathrm{e}^{-\mathrm{i} k_{1} r_{n}}\right| \chi_{m}\right\rangle\left\langle\chi_{m}\left|\mathrm{e}^{\mathrm{i} k_{0} r_{n}}\right| \chi_{i}\right\rangle \mathrm{e}^{-\mathrm{i} \omega_{m i}^{(p)} t} \\
& \times \int_{0}^{t} \mathrm{~d} t^{\prime} \exp \left(\mathrm{i}\left[\omega_{r}+\omega_{m i}^{(p)}-\omega_{0}\right] t^{\prime}\right) \mathrm{e}^{\gamma t^{\prime}} \tilde{P}\left(t^{\prime}\right) .
\end{aligned}
$$

General evaluation of this expression requires an explicit form of the response function of the monochromator. 
Below we consider the case where the energy bandwidth of the instrumental function $\hbar \Delta \omega$ is much larger than that of nuclear resonance. Therefore, the response function $\tilde{P}(t)$ decreases significantly during the characteristic time $t_{p}=1 / \Delta \omega$, which is much less than the life time of the excited nuclear state $t_{0}=1 / 2 \gamma$. For example, the typical value $\hbar \Delta \omega=1 \mathrm{meV}$ leads to an estimation $t_{p}=0.0007 \mathrm{~ns}$ whereas $t_{0}=141 \mathrm{~ns}$ for the ${ }^{57} \mathrm{Fe}$ nucleus. Then the function $\exp \left(\gamma t^{\prime}\right)$ under the integral can be approximated by unity. It is evident that within the time interval $0<t<t_{p}$ the integral in (42) increases with time and becomes constant when $t \gg t_{p}$. In this latter region we can replace the upper limit by $\infty$. Omitting also a constant phase factor, which does not influence the intensity, we obtain

$Q_{f i}\left(\omega_{0}, t\right)=\mathrm{e}^{-\gamma t} \sum_{m}\left\langle\chi_{f}\left|\mathrm{e}^{-\mathrm{i} k_{1} r_{n}}\right| \chi_{m}\right\rangle\left\langle\chi_{m}\left|\mathrm{e}^{\mathrm{i} k_{0} r_{n}}\right| \chi_{i}\right\rangle \mathrm{e}^{-\mathrm{i} \omega_{m i}^{(p)} t} P\left(\omega_{r}+\omega_{m i}^{(p)}-\omega_{0}\right)$.

Repeating the derivation as discussed above, we arrive at the following expression for the average intensity:

$\overline{I\left(\omega_{0}, t\right)}=I_{0} \exp \left(-t / t_{0}\right) I_{N} \sum_{i, f} g_{i}\left|\left\langle\chi_{f}\left|\exp \left(\mathrm{i} \boldsymbol{k}_{0} \boldsymbol{r}_{n}\right)\right| \chi_{i}\right\rangle\right|^{2} I_{M}\left(\omega_{r}+\omega_{f i}^{(p)}-\omega_{0}\right)$.

Thus within the time region $t \gg t_{p}$ the intensity decreases in an expected way, i.e. exponentially according to the lifetime of the excited nuclear state.

Equation (44) is similar to equation (31), derived above for the case of nuclear inelastic absorption. Following the same approach, we obtain

$$
\overline{I\left(\omega_{0}, t\right)}=I_{0} \exp \left(-t / t_{0}\right) I_{N} \int \mathrm{d} t^{\prime} \exp \left(-\mathrm{i}\left[\omega_{0}-\omega_{r}\right] t^{\prime}\right) F\left(\boldsymbol{k}_{0}, t^{\prime}\right) \tilde{I}_{M}\left(t^{\prime}\right) .
$$

If the lifetime of an excited nuclear state is, as we assume here, much longer than the flash of radiation transmitted by the monochromator, we can neglect the time dependence of the function $R(t)$ in (37) and replace $R(t)$ by $R(0)$. Then the intensity integrated over time (45) coincides with the approximate expression (37), obtained by the other method.

The time dependence of $R(t)$ in (37) describes an exact behaviour of the delayed radiation soon after the flash provided by the monochromator. Within this time interval the intensity of the scattered radiation increases from zero to a certain maximal value. When the flash is over, the major effect is the delayed re-emission of the excited nuclear state, which decays exponentially with time.

Equation (45) shows that the energy dependence of the incoherent nuclear inelastic scattering is identical at any given moment $t \gg t_{p}$. In practice, the acquisition system is gated during the time interval $\Delta t$ after the primary flash, and $\Delta t \gg t_{p}$. The typical value of $\Delta t$ is a few nanoseconds. So we can conclude that the variation of the gating interval influences only the average count rate without changing the shape of the energy dependence of inelastic scattering.

\section{Effect of hyperfine structure of nuclear levels}

In this section we consider a more complicated case, where nuclear levels of both ground and excited states are split by hyperfine interaction. In equations (11), (14) and further down we have to replace $\omega_{i}$ by $\omega_{i}+\Delta \omega_{\alpha}^{(i)}$, $\omega_{j}$ by $\omega_{j}+\Delta \omega_{\beta}^{(j)}$ and $\omega_{f}$ by $\omega_{f}+\Delta \omega_{\gamma}^{(f)}$, where indices $\alpha, \beta$ and $\gamma$ denote various sub-levels of hyperfine structure for the initial, intermediate and final states, respectively. Accordingly, nuclear wavefunctions have indices $\left|\phi_{i \alpha}\right\rangle$ and so on. Equation (14) for the scattering matrix becomes

$$
\begin{aligned}
M_{f i}\left(\boldsymbol{r}, t ; \boldsymbol{r}^{\prime}, t^{\prime}\right) & =\frac{\mathrm{i} \exp \left(\mathrm{i} \omega_{f \gamma, \mathrm{i} \alpha}^{(n)} t\right)}{\hbar c} \int \frac{\mathrm{d} \boldsymbol{k} \mathrm{d} \boldsymbol{k}^{\prime}}{(2 \pi)^{6}} \mathrm{e}^{\mathrm{i} k r+\mathrm{i} k^{\prime} \boldsymbol{r}^{\prime}} N_{f i}^{\gamma \alpha}\left(\boldsymbol{k}, \boldsymbol{k}^{\prime}, t-t^{\prime}\right) \\
& \times \exp \left[\mathrm{i}\left(-\omega_{j i}^{(n)}+\mathrm{i} \gamma\right)\left(t-t^{\prime}\right)\right] \mathrm{e}^{-\mathrm{i} k r_{n}(t)} \mathrm{e}^{-\mathrm{i} k^{\prime} \boldsymbol{r}_{n}\left(t^{\prime}\right)},
\end{aligned}
$$


where $\omega_{f \gamma, \mathrm{i} \alpha}=\omega_{f}+\Delta \omega_{\gamma}^{(f)}-\omega_{i}-\Delta \omega_{\alpha}^{(i)}$, and the nuclear function $N_{f i}^{\gamma \alpha}\left(\boldsymbol{k}, \boldsymbol{k}^{\prime}, t\right)$ acquires the time dependence

$N_{f i}^{\gamma \alpha}\left(\boldsymbol{k}, \boldsymbol{k}^{\prime}, t\right)=\sum_{\beta}\left\langle\phi_{f \gamma}\left|\boldsymbol{e}_{f} \hat{\boldsymbol{j}}_{n}(\boldsymbol{k})\right| \phi_{j \beta}\right\rangle\left\langle\phi_{j \beta}\left|\boldsymbol{e}_{i} \hat{\boldsymbol{j}}_{n}\left(\boldsymbol{k}^{\prime}\right)\right| \phi_{i \alpha}\right\rangle \exp \left(\mathrm{i} \Delta \omega_{j \beta, i \alpha} t\right)$,

where $\Delta \omega_{j \beta, i \alpha}=\Delta \omega_{\beta}^{(j)}-\Delta \omega_{\alpha}^{(i)}$.

Repeating the derivation discussed above, it is easy to obtain the general expression for the time dependence of intensity as

$$
I\left(\omega_{0}, t\right)=I_{0} \sum_{\alpha, \gamma} g_{\alpha}\left|\tilde{Q}_{f i}^{\gamma \alpha}\left(\omega_{0}, t\right)\right|^{2},
$$

where $g_{\alpha}$ is the statistical weight of the state $(\mathrm{i} \alpha)$, and

$\tilde{Q}_{f i}^{\gamma \alpha}\left(\omega_{0}, t\right)=\int_{0}^{t} \mathrm{~d} t^{\prime} \exp \left(\mathrm{i}\left[\omega_{0}-\omega_{r}+\mathrm{i} \gamma\right] t^{\prime}\right) N_{f i}^{\gamma \alpha}\left(\boldsymbol{k}_{1},-\boldsymbol{k}_{0}, t^{\prime}\right) L_{f i}\left(\boldsymbol{k}_{1},-\boldsymbol{k}_{0}, t^{\prime}\right) \tilde{P}\left(t-t^{\prime}\right)$.

We note that the hyperfine splitting can significantly exceed the width of the nuclear resonance. Nevertheless, the characteristic time $t_{N} \approx 1 / \omega_{N}$ of nuclear function variation is much larger than the characteristic time $t_{p}$ of the response function of the monochromator. Therefore, at $t \gg t_{p}$ we can replace the lower limit of integration by $-\infty$ and the function $\exp \left(-\gamma t^{\prime}\right) N_{f i}^{\gamma \alpha}\left(\boldsymbol{k}_{1},-\boldsymbol{k}_{0}, t^{\prime}\right)$ by its value at the upper limit $t$.

Then instead of (45), we obtain the generalized equation

$\overline{I\left(\omega_{0}, t\right)}=I_{0} \exp \left(-t / t_{0}\right) I_{N}(t) \int \mathrm{d} t^{\prime} \exp \left(-\mathrm{i}\left[\omega_{0}-\omega_{r}\right] t^{\prime}\right) F\left(\boldsymbol{k}_{0}, t^{\prime}\right) \tilde{I}_{M}\left(t^{\prime}\right)$,

where

$$
I_{N}(t)=\sum_{\alpha, \gamma} g_{\alpha}\left|N_{f i}^{\gamma \alpha}\left(\boldsymbol{k}_{1},-\boldsymbol{k}_{0}, t\right)\right|^{2}
$$

In order to obtain the space-integrated intensity of scattered radiation $I_{N}(t)$, one should perform an integration over directions of the vector $\boldsymbol{k}_{1}$. The function $I_{N}(t)$ does not depend on the resonance condition, and in particular does not depend on the value $\left(\omega_{0}-\omega_{r}\right)$. Therefore, it does not influence the energy dependence of incoherent nuclear inelastic scattering.

\section{Conclusion}

We have presented the theoretical analysis of the energy dependence and time evolution of nuclear inelastic resonant scattering of synchrotron radiation accompanied by absorption and emission of phonons in a crystal lattice. The analysis is based on Maxwell's equations and the time-dependent perturbation theory of quantum mechanics. This allows us to analyse explicitly the physical nature of the considered processes.

We use an approximation of incoherent scattering. It is justified by the small crosssection of inelastic nuclear scattering, which does not allow multiple-scattering events, and by averaging over directions of scattered quanta. Therefore, though the scattering process is coherent in general, an observation of possible interference phenomena is not permitted by conditions of measurements.

The concept of incoherent scattering significantly simplifies the analysis, because it avoids calculations of the interference, which would in any event vanish during averaging. Our results show that if measurements are performed without energy or angular analysis of scattered radiation, the dependence of its intensity on the energy of the incident radiation is identical to that of nuclear inelastic absorption. Formally, this was caused by averaging of scattering intensity over the final states of the phonon system. This allows one to apply the same technique 
for a reconstruction of the density of phonon states from scattering experiments as used in the absorption experiments.

The energy dependence of scattering is influenced by the processes of absorption and emission of phonons only during the nuclear excitation by the flash of synchrotron radiation. We assume that $\mathrm{x}$-rays emitted by various electrons in a storage ring are incoherent, and radiation from a single electron can be considered as instantaneous. This assumption is based on experimental conditions, where the intensity of scattered radiation is accumulated after many SR flashes, whereas only one photon is recorded after a particular SR flash. In the first Born approximation, the calculations are reduced to the thermal averaging of the phase factor, which depends on the nuclear coordinates at the moments of photon absorption and emission. Within this approach, the time correlation function can be calculated with a proper account for multi-phonon processes, whereas treating the multi-phonon processes in the case of coherent scattering is a significantly more complicated problem.

\section{Acknowledgment}

The work is supported by the Russian Foundation for Basic Research (project no 01-02-16508).

\section{References}

[1] Mössbauer R L 1958 Z. Phys. 151124

[2] Kagan Yu 1999 Hyperfine Interact. 123/124 83

[3] Hannon J P and Trammell G T 1999 Hyperfine Interact. 123/124 127

[4] Visscher W M 1960 Ann. Phys., NY 9194

[5] Singwi K S and Sjölander A 1960 Phys. Rev. 1201093

[6] Kagan Yu M and Iosilevskii Yu A 1962 Zh. Eksp. Teor. Fiz. 42259 (in Russian) (Engl. transl. 1962 Sov. Phys.JETP 15 182)

Kagan Yu M and Iosilevskii Yu A 1963 Zh. Eksp. Teor. Fiz. 44284 (in Russian) (Engl. transl. 1963 Sov. Phys.JETP 17 195)

[7] Chumakov A I and Sturhahn W 1999 Hyperfine Interact. 123/124 781

[8] Kohn V G, Chumakov A I and Rüffer R 1998 Phys. Rev. B 588437

[9] Sturhahn W and Kohn V G 1999 Hyperfine Interact. 123/124 369

[10] Laue M V 1960 Röntgenstrahlinterferenzen (Frankfurt)

[11] Heitler W 1944 The Quantum Theory of Radiation (New York: Oxford University Press)

[12] Chumakov A I, Barla A, Rüffer R, Metge J, Grünsteudel H F, Grünsteudel H, Plessel J, Winkelmann H and Abd-Elmeguid M M 1998 Phys. Rev. B 58254

[13] Sakurai J J 1967 Advanced Quantum Mechanics (Reading, MA: Addison-Wesley)

[14] Bürck U v 1999 Hyperfine Interact. 123/124 483

[15] Kohn V, Snigireva I and Snigirev A 2000 Phys. Rev. Lett. 852745

[16] Ternov I M 1995 Usp. Fiz. Nauk 165429 (in Russian) (Engl. transl. 1995 Sov. Phys.-Usp. 38 409)

[17] Margaritondo G 1995 J. Synchrotron Radiat. 2148

[18] Kohn V G and Chumakov A I 2000 Hyperfine Interact. 125205 